Article

\title{
An Optimal Source-Load Coordinated Restoration Method Considering Double Uncertainty
}

\author{
Ping Jiang and Qiwei Chen * \\ School of Electrical Engineering, Southeast University, Nanjing 210096, China; jping@seu.edu.cn \\ * Correspondence: chenqiwei0529@163.com; Tel.: +86-139-5193-9388
}

Received: 3 February 2018; Accepted: 27 February 2018; Published: 5 March 2018

\begin{abstract}
The security of power system restoration is severely affected by uncertain factors, especially the start-up time of generating unit and the amount of load pick-up. Solving the optimization restoration problem is challenging since it needs to determine different priorities in which units and loads are restored with the consideration of double uncertainty. Therefore, an optimal source-load coordinated restoration method that is based on information gap decision theory (IGDT) is proposed. Firstly, the time-domain restoration characteristics of black-start unit (BSU), non-black-start unit (NBSU), and load are described with analysis of double uncertainty. On this basis, a coupled multi-objective optimization model is built with double uncertainty, in which source-load coordinated restoration is realized. Then, IGDT is adopted to convert the uncertainty optimization model to a certain one with robustness, which tolerates the most uncertainty and still meets the desired requirement. Finally, the optimization model is solved by non-dominated genetic algorithm II (NSGA-II). The effectiveness and robustness of the proposed method is further illustrated through a case study based on the IEEE 39-bus system.
\end{abstract}

Keywords: power system restoration; load restoration; uncertainty multi-objective optimization; information gap decision theory

\section{Introduction}

With the continuous development of power system, the operation of the system has been more complex than ever. Due to some occasional and inevitable factors, blackouts have become a serious threat to the power system [1,2]. Besides, the security of power system has been severely affected by uncertain factors. Therefore, the investigation on source-load coordinated restoration method considering double uncertainty plays supporting role in restoration decision, which is beneficial to ensure the stability and rapidity of power system in restoration process.

The procedure of power system restoration can be divided into three stages: black-start, reconfiguration of the network and load restoration [3,4]. There is no obvious sequence as three stages are interleaved with each other. It is necessary to restore part of the important loads to balance the output of the restarted units during the black-start stage, which ensures the stable operation of the restarted units and achieves the minimum steady output as soon as possible after connecting to the grid $[5,6]$.

In recent years, theoretical investigations on unit and load restoration have attracted researchers' interests. To maximize the generating power output, the start-up of generating units need to be optimized [7]. Sun W. et al. applied mixed-integer linear programming (MILP) algorithm to identify the optimal start-up sequence of NBSUs [8]. An optimal restoration approach based on the Wide Area Measurement System (WAMS) was proposed by Nourizadeh S. et al. [9]. Gu X. et al. proposed an extended black-start restoration strategy, in view of the fact that some current black-start resources with large rated power can provide enough cranking power for more than one non-black-start unit 
(NBSU) simultaneously [10]. The feasibility of selecting microgrids as black start power was analyzed by Wu Z. et al. It adopts the Dijkstra algorithm to search for the extended black start paths [11]. Lin Z. et al. put forward a novel method using the entropy weight-based decision-making theory to evaluate and optimize the sequence of NBSUs [12]. In terms of load restoration, a DC optimal load shed recovery with transmission switching model was proposed to increase the operating capacity, when considering load demands as continuous variables [13]. In [14], a mixed-integer model that was considering discrete load pickup was proposed. An optimization model was formulated to maximize load pick-up for a given substation by Qu H. et al. [15]. Gholami A. et al. proposed a two-stage hierarchical approach to load restoration problem, which determines the optimal amount and location of load pick-up [16]. In [17], synchrophasors were used for the prediction of restorable size of load pick-up during the load restoration. Furthermore, a multi-objective optimization method coordinating unit restarting with load restoration was employed into the extended black-start by Wang D. et al. [18]. The method shows that unit and load can be effectively and coordinately restored at the black-start stage.

The aforementioned methods can yield the global optimum given that sufficient modeling details and appropriate estimations are available. However, the double uncertainty, including the start-up time of generating unit and the amount of load pick-up, severely affects the performance of restoration process, which would further reduce the applicability of classical methods. Start-up time of generating unit is closely related to the characteristics of the thermal system, which can be represented as shutting down time of the unit [19]. Besides, the amount of load pick-up is fuzzy uncertain, owing to the influence of precipitation, temperature, cold pick-up and discrete access [20]. The distribution characteristics and membership function of uncertainty are hard to obtain, result in the limitation of fuzzy random method. Nevertheless, new ideas are provided by information gap decision theory (IGDT), which can tolerate the most uncertainty and still provides the desired performance [21]. The IGDT model neither need distribution characteristics nor membership function of uncertainties. This method has been widely adopted in purchasing strategy in electricity market [22] and optimal scheduling [23].

On this basis, an optimal source-load coordinated restoration method based on IGDT is proposed in this paper, which meets the restoration requirements in the fluctuation range of double uncertainty. The time-domain restoration characteristics of black-start unit (BSU), NBSU and load are described with analysis of double uncertainty. Based on that, two objectives are considered and coupled with each other, in which the generating power output of restarted units and the weighted amount of load pick-up both are maximized. Then IGDT is adopted to convert the uncertainty multi-objective optimization model to a certain one with robustness and non-dominated genetic algorithm II (NSGA-II) is applied to solve it. More specifically, this study makes the following contributions that:

(1) A coupled multi-objective optimization model is built, which takes account of the double uncertainty in restoration process and realizes source-load coordinated restoration.

(2) IGDT is adopted to power system restoration in order to convert the uncertainty multi-objective optimization model to a certain one with robustness. The requirement of distribution characteristics and membership function of uncertainties are avoided. Moreover, the optimal solution tolerates the most uncertainty and still meets the desired requirement.

The rest of the paper is arranged as follows: Section 2 presents the double uncertainty analysis. Section 3 presents the proposed source-load coordinated restoration model considering double uncertainty. Section 4 presents the solution to the model based on IGDT and NSGA-II. A case study is shown in Section 5. The conclusions are drawn in the last section.

\section{Analysis of Double Uncertainty}

In the process of source-load coordinated restoration, the double uncertainty including the start-up time of generating unit and the amount of load pick-up severely affect restoration time, generating 
power output, and stability. Therefore, a study of double uncertainty is conducted in this section. The time-domain restoration characteristics of BSU, NBSU, and load are described with analysis of double uncertainty, which lays the foundation for later modeling.

\subsection{Uncertainty of Start-Up Time of Generating Unit}

First of all, a brief analysis of BSU $i$ is carried out. The time-domain restoration characteristic is shown in Figure 1.

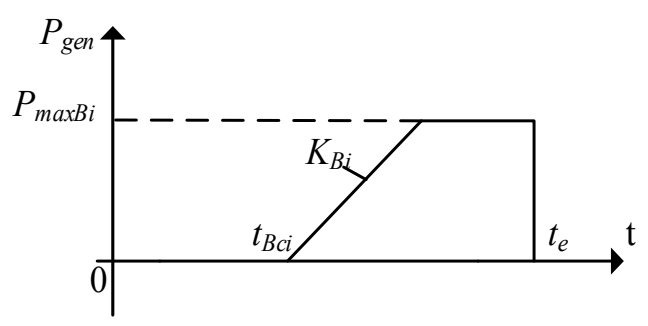

Figure 1. The time-domain restoration characteristic of BSU.

where $K_{B i}$ is the ramping rate of BSU $i$; $t_{e}$ is the entire restoration time for system; and, $t_{B c i}$ is the time when BSU $i$ begins to ramp up. BSU is not involved in optimization, owing to only one BSU in a system after blackouts. Therefore, suppose $t_{B c i}=0$ can reduce complexity in later modeling. The generating power output function $P_{B S U i}(t)$ of BSU $i$ can be expressed as:

$$
P_{B S U i}(t)= \begin{cases}K_{B i} t & 0<t<\frac{P_{\max B i}}{K_{B i}} \\ P_{\max B i} & \frac{P_{\max B i}}{K_{B i}}<t\end{cases}
$$

where $P_{\max B i}$ is the maximum generating power output of BSU $i$.

In contrast, a brief analysis of NBSU $j$ affected by uncertainty is carried out. The time-domain restoration characteristic is shown in Figure 2.

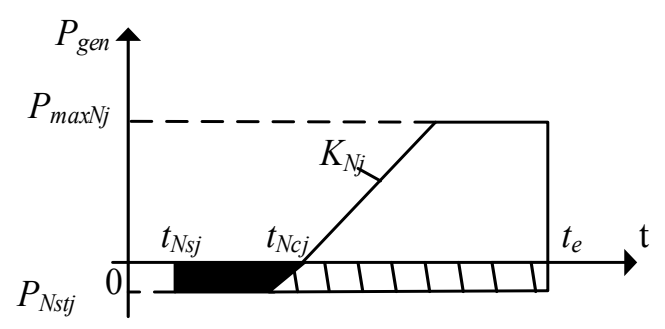

Figure 2. The time-domain restoration characteristic of NBSU.

NBSU $j$ is being cranked, but not paralleled with system from $t_{N s j}$ to $t_{N c j}$. After $t_{N c j}$, NBSU $j$ begins to ramp up. Obviously, the length of time when NBSU $j$ is being cranked but not paralleled with system $\left(t_{N s j} \sim t_{N c j}\right)$ is affected by the temperature and pressure of the thermal system, which is positively related to the shutting down time of the NBSU $j\left(0 \sim t_{N s j}\right)$. Thus, the start-up time of NBSU $j$ is uncertain and $\overline{t_{N c j}}$ is used to describe the uncertainty time when NBSU $j$ begins to ramp up. $K_{B i}$ is ramping rate of NBSU $j ; P_{\operatorname{maxN} j}$ is the maximum generating power output of NBSU $j ; P_{N s t j}$ is the 
cranking power requirement of NBSU $j$. The generating power output function $P_{\mathrm{NBSU}}(t)$ of NBSU $j$ can be expressed as:

$$
P_{N B S U j}(t)= \begin{cases}0 & 0<t<t_{N s j} \\ -P_{N s t j} & t_{N s j}<t<\overline{t_{N c j}} \\ K_{N j}\left(t-\overline{t_{N c j}}\right) & \overline{t_{N c j}}<t<\frac{P_{\max j j}}{K_{N j}}+\overline{t_{N c j}} \\ P_{\max N j} & \frac{P_{\operatorname{maxNj}}}{K_{N j}} \overline{{t_{N c j}}_{N c}}<t\end{cases}
$$

Although the distribution characteristics and membership function of $\overline{t_{N c j}}$ are hard to obtain, the fluctuation range of the time from $t_{N s j}$ to $\overline{t_{N c j}}$ is available by dispatcher. Therefore, $\overline{t_{N c j}}$ can be expressed as:

$$
\left\{\begin{array}{l}
\overline{t_{s u j}}=\overline{t_{N c j}}-t_{N s j} \\
\overline{t_{s u j}} \in U\left(a_{1}, t_{E s u j}\right) \\
U\left(a_{1}, t_{E s u j}\right)=\left\{\overline{t_{s u j}}:\left|\left(\overline{t_{s u j}}-t_{E s u j}\right) / t_{E s u j}\right| \leq a_{1}\right\}
\end{array}\right.
$$

where $\overline{t_{s u j}}$ is also uncertain, which represents the length of time when NBSU $j$ is being cranked. $t_{E s u j}$ is predicted length of that time based on experience and historical data, and $a_{1}$ is the uncertainty radius of $\overline{t_{s u j}}$. In other words, values of $\overline{t_{s u j}}$ whose deviation from $t_{E s u j}$ is nowhere greater than $a_{1} t_{E s u j}$.

\subsection{Uncertainty of Amount of Load Pick-Up}

The time-domain restoration characteristic about feeder $k$ of load $l$ is analyzed and shown in Figure 3.

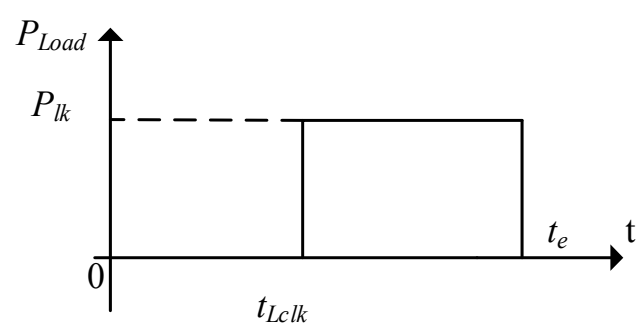

Figure 3. The time-domain restoration characteristic of load.

The time-domain restoration characteristic of load is simplified as a step function. Owing to the influence of precipitation, temperature, cold pick-up, and discrete access, $P_{l k}$ is uncertain, which represents the amount of load pick-up in feeder $k$ of load $l$. Therefore, $\overline{P_{l k}}$ is used to describe the uncertainty amount of load pick-up in feeder $k$ of load $l$. $t_{L c l k}$ is the time when feeder $k$ of load $l$ is restored. The function of load pick-up $P_{\text {Loadlk }}(t)$ can be expressed as:

$$
P_{\text {Loadlk }}(t)= \begin{cases}0 & 0<t<t_{\text {Lclk }} \\ \overline{P_{l k}} & t_{\text {Lclk }}<t\end{cases}
$$

Similarly, in order to accurately express the uncertainty amount of load pick-up for later modeling, $\overline{P_{l k}}$ can be expressed as:

$$
\left\{\begin{array}{l}
\overline{P_{l k}} \in U\left(a_{2}, P_{E l k}\right) \\
U\left(a_{2}, P_{E l k}\right)=\left\{\overline{P_{l k}}:\left|\left(\overline{P_{l k}}-P_{E l k}\right) / P_{E l k}\right| \leq a_{2}\right\}
\end{array}\right.
$$

where $P_{E l k}$ is predicted amount of load pick-up in feeder $k$ of load $l ; a_{2}$ is the uncertainty radius of $\overline{P_{l k}}$. 


\section{Source-Load Coordinated Restoration Model Considering Double Uncertainty}

The main task of source-load coordinated restoration is to optimize the restoration sequence of NBSUs and loads. The interval between two units begin to restart is set as a time-step, in which important loads are coordinately restored to absorb excessive active power that is generated. The source-load coordinated restoration model is formulated as a multi-objective optimization problem; the optimization model, including objective function and constraints, are described in detail in this section.

\subsection{Objective Function}

\subsubsection{Objective of Unit Restoration}

The maximum generating power output of restarted units can be expressed as:

$$
f_{1}=\max \left(\sum_{i=1}^{n} \int_{0}^{t_{e}} P_{B S U i}(t)+\sum_{j=1}^{m} \int_{0}^{t_{e}} P_{\text {NBSUj }}(t)\right)
$$

where $n$ is the number of BSUs in the system; $m$ is the number of NBSUs in the system.

It is not easy to solve the objective function containing the integral process. Therefore, the calculation of objective function is converted to that of the maximum area that is encircled by time-domain characteristics curve of units and $X$ axis in this paper. The computational efficiency is obviously improved, which only needs simple algebraic operations. As mentioned above, the time when BSUs begin to ramp is the same as that when the system starts to restore. The first part in Equation (6) can be ignored, as it represents the generating output of BSUs, which is constant.

The second part in Equation (6) can be expressed as the area of white trapezoid in Figure 2. The following equation represents the generating power output of NBSU $j$.

$$
\frac{1}{2} P_{N B S U j}(t)\left[\left(t-\overline{t_{N c j}}\right)+\left(t-\frac{P_{N B S U j}(t)}{K_{N j}}-\overline{t_{N c j}}\right)\right]-\left(t-t_{N s j}\right) P_{N s t j}
$$

where $t_{N s j}$ is a decision variable to be solved. The maximum generating output of all the NBSUs in system can be expressed as:

$$
f_{1}=\max \sum_{j=1}^{m} P_{N B S U j}(t) \cdot\left(t-\overline{t_{s u j}}-2 t_{N s j}-\frac{P_{N B S U j}(t)}{2 K_{N j}}\right)-\sum_{j=1}^{m}\left(t-t_{N s j}\right) P_{N s t j}
$$

\subsubsection{Objective of Load Restoration}

While the NBSUs are being restored, the important load should be taken into account to balance active power and accelerate restoration process. The maximum weighted amount of load pick-up can be expressed as:

$$
f_{2}=\max \sum_{l=1}^{N_{k}} \sum_{k=1}^{N_{p}} \omega_{l k} P_{\text {Loadlk }}(t)
$$

where $N_{k}$ is the number of load nodes in the system; $N_{p}$ is the number of feeders belonging to load l. $\omega_{l k}$ is the weight of load in feeder $k$ of load $l$. The weight is determined by Analytic Hierarchy Process with the type of load by Wang D. et al. [18], or even set according to the need and preference of the dispatcher.

In summary, two objectives are coupled with each other through time variable $t$. The objective function of the model is as follow:

$$
\operatorname{Max}\left(f_{1}, f_{2}\right)
$$




\subsection{Constraints}

\subsubsection{Cranking Power Constraint}

The sum of cranking power for NBSUs to be restarted and the active power of loads pick-up must be less than the available generating power output provided by restarted units. Therefore, the cranking power constraint to NBSU $j$ is as follows:

$$
\sum_{i=1}^{n} P_{\text {BSUi }}(t)+\sum_{j=1}^{m} P_{\text {NBSUj }}(t)-\sum_{l=1}^{N_{k}} \sum_{k=1}^{N_{p}} P_{\text {Loadlk }}(t)-\sum_{j=1}^{m}\left(1-u_{j}(t)\right) \cdot u_{j}(t+\Delta t) P_{N s t j} \geq 0
$$

There are four parts in Equation (11). The first and second parts represent generating power output provided by restored BSUs and NBSUs, respectively. The third part is the amount of load pick-up and the last part is the cranking power for NBSU $j$ to be restarted. $u_{j}(t)$ indicates the state of NBSU $j$ at time $t$. Only when $u_{j}(t)=0$ and $u_{j}(t+\Delta t)=1$, NBSU $j$ begins to ramp up. NBSU should keep operating state once restarted. Assuming that NBSU $j$ no longer shuts down after restarted, it can be expressed as follow:

$$
u_{j}(t)<u_{j}(t+\Delta t)
$$

\subsubsection{Maximum Power Constraint to Load Pick-Up at One Time}

The maximum power of load to be restored at one time must meet the requirements of the system stability; otherwise, the excessive voltage drop and frequency deviation may lead to failure of system restoration. The maximum power constraint to load pick-up at one time is given by:

$$
\overline{P_{l k}} \leq \Delta f_{\max }\left(\sum_{i=1}^{n} \frac{P_{B S U n i}}{f_{d i}}+\sum_{j=1}^{m} \frac{u_{j}(t) P_{N B S U n j}}{f_{d j}}\right)
$$

where $P_{B S U n i}$ and $P_{N B S U n j}$ are the active rated power of BSU $i$ and NBSU $j$, respectively; $\Delta f_{\max }$ is the permitted frequency drop; $f_{d i}$ and $f_{d j}$ are the frequency response rate of BSU $i$ and NBSU $j$, which can be obtained from reference [24].

\subsubsection{Reactive Power Constraint}

The reactive power generated by restoration paths must be less than the possible maximum reactive power that is absorbed by BSUs; otherwise, the excessive reactive power may lead to sustained overvoltage and transformer over excitation. The reactive power constraint is given, as follows:

$$
\sum_{p=1}^{N_{p}} Q_{p}<\sum_{i=1}^{n} Q_{B i \max }
$$

where $N_{p}$ is the number of transmission lines in the restoration paths; $Q_{p}$ is the charging reactive power of the transmission line $p, Q_{B i m a x}$ is the maximum reactive power absorbed by BSU $i$.

Besides, the charging reactive power of restoration paths may also lead to the self-excitation of BSU. In order to avoid this situation, the self-excitation constraint to BSU is expressed as:

$$
\sum_{p=1}^{N_{p}} Q_{p}<\sum_{i=1}^{n} K_{Q i} S_{B i}
$$

where $K_{\mathrm{Q} i}$ and $S_{B i}$ are the short circuit ratio and the rated power of BSU $i$, respectively. 


\subsubsection{System Operating Constraint}

The system operating constraint includes voltage and reactive power output of units.

$$
\left\{\begin{array}{l}
Q_{G i \min } \leq Q_{G i} \leq Q_{G i \max } \\
Q_{G j \min } \leq Q_{G j} \leq Q_{G j \max } \\
V_{i \min } \leq V_{i} \leq V_{i \max } \\
V_{j \min } \leq V_{j} \leq V_{j \max } \\
V_{l \text { min }} \leq V_{l} \leq V_{l \max }
\end{array}\right.
$$

where $Q_{G i}$ and $Q_{G j}$ are the reactive power output of BSU $i$ and NBSU $j$, respectively. $Q_{G i m i n}$ and $Q_{G j \min }$ are the minimum reactive power output of BSU $i$ and NBSU $j$, respectively. $Q_{G i m a x}$ and $Q_{G j \max }$ are the maximum reactive power output of BSU $i$ and NBSU $j$, respectively. $V_{i}, V_{j}$ and $V_{l}$ are voltage amplitude of BSU $i, \mathrm{NBSU} j$ and load $l$, respectively. $V_{i \max }, V_{j \max }$ and $V_{l \max }$ are the permitted voltage upper limits of BSU $i, \mathrm{NBSU} j$ and load $l$, respectively. $V_{i \min }, V_{j \min }$ and $V_{l \min }$ are the permitted voltage lower limits of BSU $i$, NBSU $j$ and load $l$, respectively.

\subsubsection{Maximum Critical Hot-Start Time Constraint}

To make the unit restarted within the maximum critical time, the maximum critical hot-start limitation of NBSU must be longer than the time when the NBSU begins to ramp up. Therefore, the maximum critical hot-start constraint to NBSU is given by:

$$
0<t_{N s j}<t_{C H}
$$

where $t_{C H}$ is the maximum critical hot-start limitation of NBSU.

\section{The Solution to Source-Load Coordinated Restoration Model Considering Double Uncertainty}

IGDT is adopted to convert uncertainty multi-objective optimization model to a certain one, which tolerates the most uncertainty and still meets the desired requirement. When compared with the fuzzy random method, IGDT neither needs distribution characteristics nor membership function of uncertainties. Finally, the specific restoration scheme can be solved by NSGA-II.

\subsection{Information Gap Decision Theory}

IGDT can build a robust model based on different requirements owing to the different preferences of decision-maker. The robustness is defined as the immunity of the minimum requirement satisfaction at presence of uncertain parameters [22].

For a given uncertainty optimization model:

$$
\begin{cases}\max B(\bar{X}, d) & \\ \text { s.t. } & H(\bar{X}, d)=0 \\ & G(\bar{X}, d) \leq 0\end{cases}
$$

where $\bar{X}$ is the uncertainty set describing the uncertain input parameters. $d$ is the decision variables set. $B(\bar{X}, d)$ represents the optimization objective. $H(\bar{X}, d)=0$ and $G(\bar{X}, d) \leq 0$ are quality and inequality constrains, respectively.

The uncertainty is defined as the distance between what is known and what may happen in reality [22]. Therefore, the envelope bound model is used to represent the prior information about the uncertain parameters $\bar{X}$, as follows [22]:

$$
\left\{\begin{array}{l}
\bar{X} \in U\left(a, X_{E}\right) \\
U\left(a, X_{E}\right)=\left\{\bar{X}:\left|\left(\bar{X}-X_{E}\right) / X_{E}\right| \leq a\right\}
\end{array}\right.
$$


where $X_{E}$ is the predicted value of the uncertain parameters $\bar{X} ; a$ is the uncertainty radius of $\bar{X}$; $U\left(a, X_{E}\right)$ is the set of all values of $\bar{X}$ whose deviation from $X_{E}$ is nowhere greater than $a \bar{X}$.

The uncertainty optimization model of Equation (18) can be converted to the certainty model based on the predicted value of the uncertain parameters $\bar{X}$ as follows:

$$
\begin{cases}\max B\left(X_{E}, d\right) & \\ \text { s.t. } & H\left(X_{E}, d\right)=0 \\ & G\left(X_{E}, d\right) \leq 0\end{cases}
$$

By solving Equation (20), the basic value of objective function is $B_{0}$. However, it is obvious that the optimal solution of Equation (20) is not reliable while the value of $\bar{X}$ may be different from the predicted value $X_{E}$. In order to guarantee the optimization effect, decision maker sets a minimum requirement $B^{\prime}$ as not surpassing a predefined limit.

$$
B^{\prime}=(1-\delta) B_{0} \quad \delta \in[0,1)
$$

where $\delta$ is a deviation factor, which is the degree of deviation between minimum requirement and the optimal solution of the certainty model. The more $\delta$ is, the bigger value of uncertainty radius of $\bar{X}$ can be tolerated.

Based on these, the uncertainty optimization model of Equation (18) can be finally converted to solve the maximum value of uncertainty radius of $\bar{X}$ when the minimum requirement is always satisfied. The robust model can be expressed as:

$$
\begin{cases}\max a & \\ \text { s.t. } & \min B\left(X_{E}, d\right) \geq B^{\prime} \\ & B^{\prime}=(1-\delta) B_{0} \\ & H\left(X_{E}, d\right)=0 \\ & G\left(X_{E}, d\right) \leq 0\end{cases}
$$

The decision scheme of Equation (22) can tolerate the maximum value of uncertainty radius of $\bar{X}$ and meet the minimum requirement. Owing to the uncertainty set $\bar{X}$ in the model has been replaced by predicted value $X_{E}$, the original objective function and constraints need to be modified to correspond with the actual requirements.

\subsection{Source-Load Coordinated Restoration Model Based on IGDT}

The uncertainty optimization model and envelope bound model of double uncertainty have been established above. Therefore, the maximum generating power output $B_{1}$ and the weighted amount of load pick-up $B_{2}$ are calculated by Equations (8) and (9), based on the predicted value $t_{E s u j}$ and $P_{E l k}$. Dispatcher set $B_{c}$ and $B_{d}$ as the minimum requirements of generating output and the amount of load pick-up, respectively.

$$
\left\{\begin{array}{l}
B_{c}=\left(1-\delta_{1}\right) B_{1} \\
B_{d}=\left(1-\delta_{2}\right) B_{2}
\end{array}\right.
$$

According to the idea of IGDT, two objective functions are converted to constraints in a new robust model, as follows:

$$
\begin{gathered}
\min \left[\sum_{j=1}^{m} P_{\text {NBSUj }}(t) \cdot\left(t-\overline{t_{s u j}}-2 t_{N s j}-\frac{P_{N B S U j}(t)}{2 K_{N j}}\right)-\sum_{j=1}^{m}\left(t-t_{N s j}\right) P_{N s t j}\right] \geq B_{c} \\
\min \sum_{l=1}^{N_{k}} \sum_{k=1}^{N_{p}} \omega_{l k} P_{\text {Loadlk }}(t) \geq B_{d}
\end{gathered}
$$


The generating power output is minimal when the output of each NBSU is the minimum. Therefore, Equation (24) should be modified as:

$$
\min \left(\sum_{j=1}^{m} P_{N B S U j}(t) \cdot\left(t-\left(1+\alpha_{1}\right) t_{E s u j}-2 t_{N s j}-\frac{P_{N B S U j}(t)}{2 K_{N j}}\right)-\sum_{j=1}^{m}\left(t-t_{N s j}\right) P_{N s t j}\right) \geq B_{C}
$$

The weighted amount of load pick-up is minimal when the amount of load restored in each feeder is the minimum. Therefore, Equation (25) should be modified as:

$$
\min \sum_{l=1}^{N_{k}} \sum_{k=1}^{N_{p}} \omega_{l k} P_{E l k}\left(t-t_{c l k}\right)\left(1-\alpha_{2}\right) \geq B_{d}
$$

Besides, the constraints also need to be modified.

Similarly, Equation (11) should be modified as the cranking power constraint to NBSU $j$ when the output of each NBSU is the minimum and the amount of load that is restored in each feeder is the maximum.

$$
\sum_{i=1}^{n} P_{\text {BSUi }}(t)+\sum_{j=1}^{m}\left(1-\alpha_{1}\right) P_{\text {NBSUj }}(t)-\sum_{l=1}^{N_{k}} \sum_{k=1}^{N_{p}}\left(1+\alpha_{2}\right) P_{\text {Loadlk }}(t)-\sum_{j=1}^{m}\left(1-u_{j}(t)\right) \cdot u_{j}(t+\Delta t) P_{\text {Nstj }} \geq 0
$$

Equation (13) should be modified as maximum power constraint to load pick-up at one time when the amount of load restored in each feeder is the maximum.

$$
\left(1+\alpha_{2}\right) P_{E l k} \leq \Delta f_{\max }\left(\sum_{i=1}^{n} \frac{P_{B S U n i}}{f_{d i}}+\sum_{j=1}^{m} \frac{u_{j}(t) P_{N B S U n j}}{f_{d j}}\right)
$$

Equations (12) and (14)-(17) do not need further modification, owing to no relationship with double uncertainty. In summary, source-load coordinated restoration model based on IGDT can be expressed as:

$$
\begin{cases}\max & \alpha_{1}, \alpha_{2} \\ \text { s.t. } & 0 \leq \alpha_{1} \leq 1,0 \leq \alpha_{2} \leq 1 \\ & \text { Equations (12) and (14) to Equations (17) and (26) to Equation (29) }\end{cases}
$$

Source-load coordinated restoration scheme calculated by this model is robust to double uncertainty. More specifically, the restoration scheme can guarantee that the generating power output and the amount of load pick-up are no less than $\left(1-\delta_{1}\right) B_{1}$ and $\left(1-\delta_{2}\right) B_{2}$ when the length of cranking time fluctuates within $\left(1-a_{1}, 1+a_{1}\right)$ and the amount of load pick-up fluctuates within $\left(1-a_{2}, 1+a_{2}\right)$.

\subsection{The Solution to Source-Load Coordinated Restoration Model Based on IGDT}

NSGA-II which is one of the most effective and efficient algorithms for solving multi-objective problems is applied to solve the certain model with robustness [25]. Its main characteristics are the following:

(1) Two fitness functions, given by $a_{1}$ and $a_{2}$

(2) Integer codification: each individual is described by the number of $s(s=m+l \times k)$ variables that are describing the time when NBSUs start to be cranked and loads are restored. Although time is not continuous and integer, Haffner $S$ built conversion that can transform the codified variables back to the original variables [26], as follows:

$$
x_{i}=x_{i \min }+y_{i} \Delta x_{i} \quad 0 \leq y_{i} \leq \frac{x_{i \max }-x_{i \min }}{\Delta x_{i}}, y_{i} \in Z
$$


where $x_{i}$ is original variable, $x_{i \max }$ and $x_{i \min }$ are upper and lower values of $x_{i} . y_{i}$ is integer variable. This paper set $1 \mathrm{~min}$ as $\Delta x_{i}$.

(3) Use of non-dominated sorting and crowding distance [27].

Based on NSGA-II algorithm, the specific solving progress shown in Figure 4 is as follows:

(1) Input basic system data.

(2) Set up the deviation factors $\delta_{1}$ and $\delta_{2}$, basic parameters of NSGA-II including number of individuals in the population $n_{p o p}$, maximum number of generation $g_{\max }$, crossover probability $p_{c}$, mutation probability $p_{m}$.

(3) Generate $3 n_{p o p}$ individuals randomly and among them select $n_{p o p}$ with distinct characteristics and constraints satisfaction as parent population $P_{t}$.

(4) Create offspring population $Q_{t}$ from $P_{t}$ by using the tournament selection, crossover and mutation operators.

(5) Combine $Q_{t}$ and $P_{t}$ to create intermediate population $R_{t}=P_{t} \cup Q_{t}$. Check constraint conditions of individuals and punish them by subtracting a large number on the fitness values $a_{1}$ and $a_{2}$ both.

(6) Perform a non-dominated sorting and crowding distance calculation to $R_{t}$ and select the first $n_{p o p}$ individuals as new parent population $P_{t+1}$.

(7) Check whether the result has reached the maximum number of iterations. If not, turn to (4), else turn to (8).

(8) Output the Pareto-optimal front.

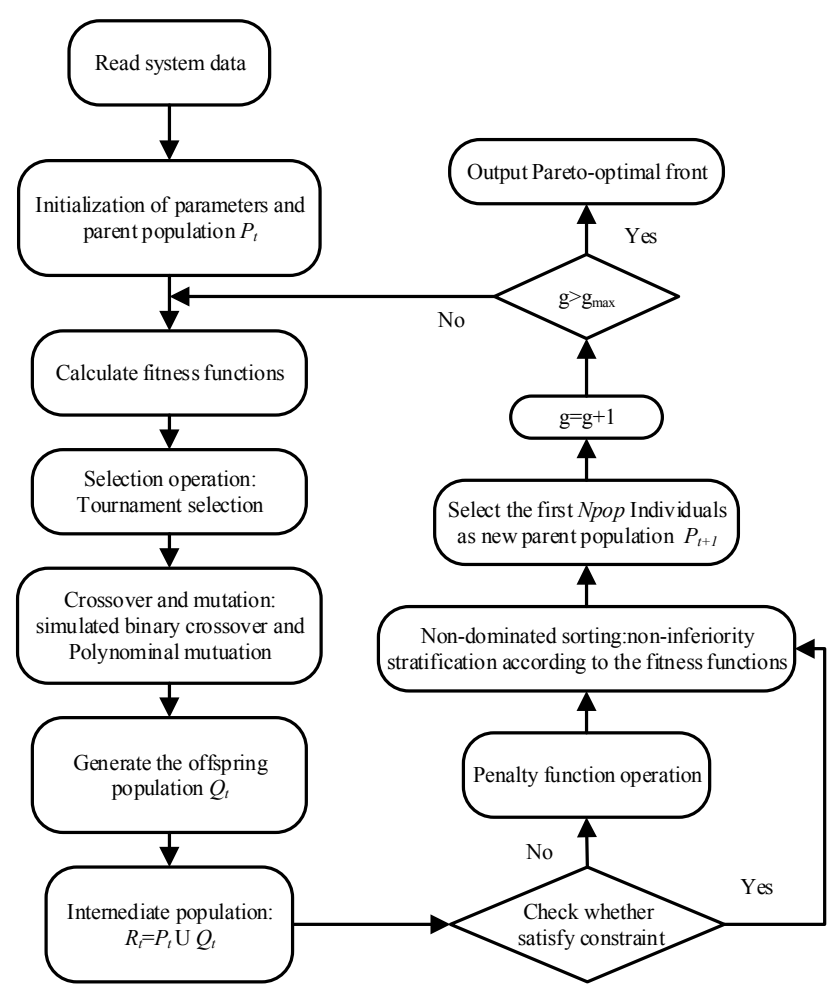

Figure 4. The flowchart of solution to source-load coordinated restoration model based on information gap decision theory (IGDT).

\section{Case Study}

The IEEE 39-bus shown in Figure 5 is used as a case study to verify the effectiveness of the proposed method. The optimization program was developed with the programming tool of 
MATLAB. Suppose that the unit at bus 33 is used as the BSU, with rated power $600 \mathrm{MW}, \cos \varphi=0.9$, $Q_{B \max }=0.3 S_{N}, K_{Q}=0.45$, and it is successfully restarted at the instant $0 \mathrm{~h}$ as the beginning time of the system restoration. The rest buses are NBSUs and loads to be restored. All of the units are subjected to a maximum critical hot start time of $1.5 \mathrm{~h}$. The numbers and parameters of the units are assumed as that in Table 1. The parameters of the loads are assumed as that in Table 2. The feeder of load is represented as $X(y)$, in which $X$ is the node number and $y$ is the feeder number. The power factors for all the loads are set to 0.8 .

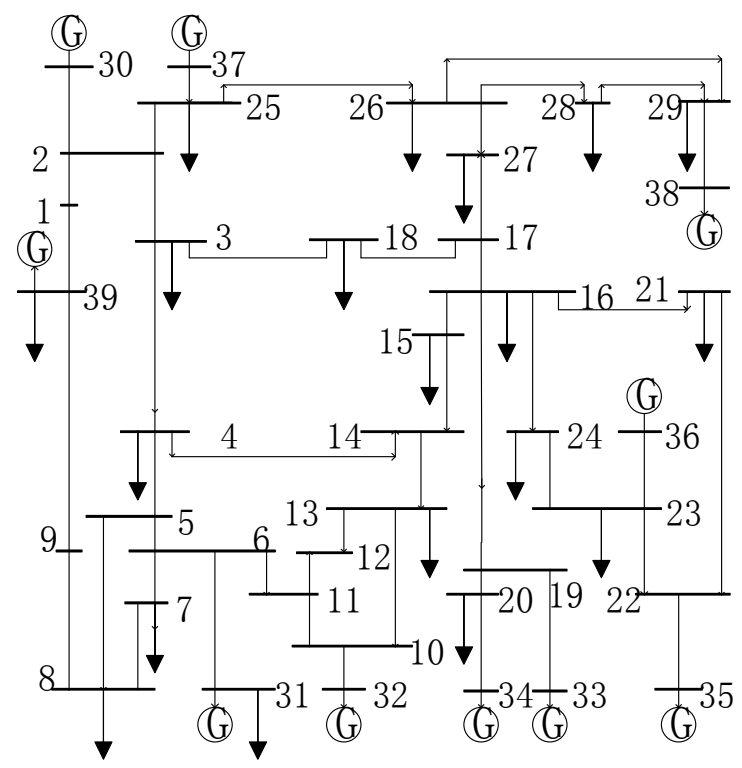

Figure 5. New England 10-unit 39-bus power system.

Table 1. The parameters of the units.

\begin{tabular}{ccccc}
\hline Unit & $\begin{array}{c}\text { Rated } \\
\text { Power/MW }\end{array}$ & $\begin{array}{c}\text { Cranking } \\
\text { Power/MW }\end{array}$ & $\begin{array}{c}\text { Ramping } \\
\text { Rate/MW/h }\end{array}$ & $\begin{array}{c}\text { Predicted } \\
\text { Cranking Time/h }\end{array}$ \\
\hline 30 & 350 & 17.5 & 127.27 & 0.67 \\
31 & 600 & 30.0 & 160.00 & 0.50 \\
32 & 250 & 12.5 & 108.23 & 0.67 \\
33 & 600 & 0.0 & 260.00 & 0.00 \\
34 & 300 & 15.0 & 179.64 & 1.10 \\
35 & 250 & 12.5 & 149.70 & 0.83 \\
36 & 330 & 16.5 & 120.00 & 1.10 \\
37 & 320 & 16.0 & 160.00 & 1.00 \\
38 & 300 & 15.0 & 200.00 & 0.83 \\
39 & 300 & 15.0 & 179.64 & 0.83 \\
\hline
\end{tabular}

The parameters of NSGA-II are set as follows: the number of individuals in the population is 200 , maximum number of generation is 20 , crossover probability is 0.8 and the mutation probability is 0.1 . Besides, $\Delta x_{i}=1 \mathrm{~min}$. The entire restoration time for system is $2 \mathrm{~h}$. The charging time for each transmission line is $2 \mathrm{~min}$. 
Table 2. The parameters of the loads.

\begin{tabular}{cccccc}
\hline Load & $\begin{array}{c}\text { Predicted } \\
\text { Amount/MW }\end{array}$ & Weight & Load & $\begin{array}{c}\text { Predicted } \\
\text { Amount/MW }\end{array}$ & Weight \\
\hline $3(1)$ & 16.1 & 0.5400 & $20(4)$ & 17 & 0.5012 \\
$3(2)$ & 16.1 & 0.5260 & $21(1)$ & 13.7 & 0.5496 \\
$4(1)$ & 12.5 & 0.5556 & $21(2)$ & 13.7 & 0.5276 \\
$4(2)$ & 12.5 & 0.5572 & $23(1)$ & 24.7 & 0.5696 \\
$4(3)$ & 12.5 & 0.5204 & $24(1)$ & 15.4 & 0.5468 \\
$4(4)$ & 12.5 & 0.5568 & $24(2)$ & 15.4 & 0.5792 \\
$7(1)$ & 23.3 & 0.4820 & $25(1)$ & 22.4 & 0.5000 \\
$8(1)$ & 17.4 & 0.4832 & $26(1)$ & 13.9 & 0.5356 \\
$8(2)$ & 17.4 & 0.5836 & $27(1)$ & 14.0 & 0.5884 \\
$8(3)$ & 17.4 & 0.5016 & $27(2)$ & 14.0 & 0.5272 \\
$12(1)$ & 8.5 & 0.5728 & $28(1)$ & 20.6 & 0.4728 \\
$15(1)$ & 16.0 & 0.5908 & $29(1)$ & 14.1 & 0.5224 \\
$15(2)$ & 16.0 & 0.5480 & $29(2)$ & 14.1 & 0.4940 \\
$16(1)$ & 10.9 & 0.5560 & $31(1)$ & 9.2 & 0.5800 \\
$16(2)$ & 10.9 & 0.5472 & $31(2)$ & 9.2 & 0.5236 \\
$16(3)$ & 10.9 & 0.5612 & $39(1)$ & 22.0 & 0.5360 \\
$18(1)$ & 15.8 & 0.5836 & $39(2)$ & 22.0 & 0.5756 \\
$20(1)$ & 17 & 0.5184 & $39(3)$ & 22.0 & 0.4800 \\
$20(2)$ & 17 & 0.5208 & $39(4)$ & 22.0 & 0.5656 \\
$20(3)$ & 17 & 0.5496 & $39(5)$ & 22.0 & 0.5480 \\
\hline
\end{tabular}

\subsection{Result of Source-Load Coordinated Restoation}

Firstly, the maximum generating power output $B_{1}$ and the weighted amount of load pick-up $B_{2}$ are calculated by Equations (8) and (9) based on the predicted value $t_{E s u j}$ and $P_{E l k}$. The unique solution can be obtained, in which the generating power output is $797.25 \mathrm{MW}$, the weighted amount of load pick-up is 343.23. The units and loads coordinately restored from $0 \mathrm{~min}$ to $85 \mathrm{~min}$. After $85 \mathrm{~min}$, only loads are restored further. The source-load coordinated restoration scheme without considering double uncertainty is shown in Table 3. The interval between two units begin to restart is set as a time-step.

Table 3. The source-load coordinated restoration scheme without considering double uncertainty.

\begin{tabular}{ccccc}
\hline Time-Step & Time/min & Unit Restoration & Load Restoration & $\begin{array}{c}\text { Amount of Load } \\
\text { Pick-Up/MW }\end{array}$ \\
\hline 1 & $0-23$ & 31 & $8(2), 15(1), 18(1), 27(1)$ & 62.8 \\
2 & $23-36$ & 38 & $24(2), 31(1), 39(2)$ & 46.6 \\
3 & $36-47$ & 39 & $23(1), 16(3)$ & 35.6 \\
4 & $47-54$ & 34 & $20(3)$ & 17.0 \\
4 & $54-61$ & 37 & $4(4), 39(4)$ & 34.5 \\
5 & $61-65$ & 30 & $12(1)$ & 8.5 \\
7 & $65-73$ & 35 & $4(2), 16(1), 39(5)$ & 45.4 \\
8 & $73-77$ & 36 & $4(1)$ & 12.5 \\
9 & $77-85$ & 32 & $15(2), 16(2), 21(1)$ & 40.6 \\
\hline
\end{tabular}

Then, double uncertainty is considered by setting the deviation factors $\delta_{1}$ and $\delta_{2}$ to determine minimum requirements $B_{c}$ and $B_{d}$. For example, $\delta_{1}$ is assumed as 0.03 and $\delta_{2}$ is assumed as 0.2 . Through solving the multi-objective optimization model that is built in this paper, the Pareto-optimal front can be obtained, as shown in Figure 6. 


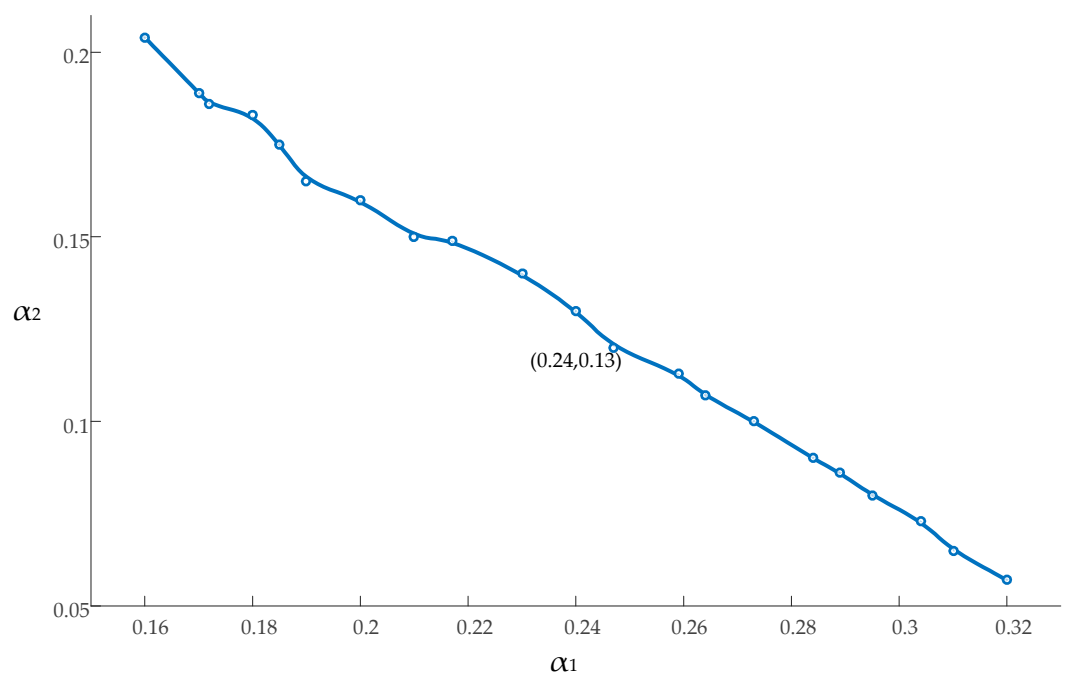

Figure 6. Pareto-optimal front.

The bigger values of uncertainty radius $a_{1}$ and $a_{2}$ are, the more robust restoration process is. In order to achieve the balance between $a_{1}$ and $a_{2}$, the midpoint of Pareto-optimal $(0.24,0.13)$ is selected, the corresponding source-load coordinated restoration scheme is shown in Table 4.

Table 4. The source-load coordinated restoration scheme considering double uncertainty.

\begin{tabular}{ccccc}
\hline Time-Step & Time/min & Unit Restoration & Load Restoration & $\begin{array}{c}\text { Amount of Load } \\
\text { Pick-Up/MW }\end{array}$ \\
\hline 1 & $0-23$ & 31 & $8(2), 31(1), 18(1), 27(1)$ & 56.4 \\
2 & $23-34$ & 39 & $15(1), 16(3)$ & 26.9 \\
3 & $34-43$ & 38 & $39(2)$ & 22.0 \\
4 & $43-49$ & 30 & $12(1)$ & 8.5 \\
4 & $49-56$ & 34 & $39(4)$ & 22.0 \\
5 & $56-64$ & 35 & $23(1), 24(2)$ & 40.1 \\
7 & $64-70$ & 36 & $16(1), 16(2)$ & 21.8 \\
8 & $70-78$ & 32 & $4(1), 4(2), 4(4)$ & 12.5 \\
9 & $78-84$ & 37 & $39(5)$ & 40.6 \\
\hline
\end{tabular}

The amount of load pick-up is 257.27 MW to balance the active power in system, accounting for eight percent of the generating output when the system is in normal operation.

\subsection{Analysis of Optimization Characteristics}

In order to further investigate the optimization characteristics of this method, the load restoration and unit restoration are optimized with the consideration of uncertainty separately. The generating power output and the weighted amount of load pick-up are compared under different deviation factors.

Firstly, the optimization characteristic of unit restoration is investigated. $\delta_{2}$ is assumed as 0 , $\delta_{1}$ is changed to determine the minimal requirement of generating power output. The midpoint of Pareto-optimal is selected to analyze. The values of uncertainty radius $a_{1}$ and corresponding unit restoration scheme are shown in Table 5 . It can be seen that the generating power output is decreasing with the increase of the deviation factor $\delta_{1}$. 
Table 5. The unit restoration scheme considering double uncertainty.

\begin{tabular}{ccccc}
\hline$\delta_{1}$ & $\boldsymbol{a}_{\mathbf{1}}$ & \multicolumn{2}{c}{ Unit Restoration } & $\begin{array}{c}\text { Generating Power } \\
\text { Output/MW }\end{array}$ \\
\hline 0.03 & \multirow{2}{*}{0.24} & $\begin{array}{c}\text { Sequence } \\
\text { Time } / \mathrm{min}\end{array}$ & $\begin{array}{l}31,39,38,30,34,35,36,32,37 \\
23,34,43,49,56,64,70,78,84\end{array}$ & 774.59 \\
\hline \multirow{2}{*}{0.06} & \multirow{2}{*}{0.53} & $\begin{array}{c}\text { Sequence } \\
\text { Time/min }\end{array}$ & $\begin{array}{l}31,32,39,36,30,34,35,37,38 \\
23,32,43,49,55,61,67,73,85\end{array}$ & 750.39 \\
\hline \multirow{2}{*}{0.09} & \multirow{2}{*}{0.77} & $\begin{array}{c}\text { Sequence } \\
\text { Time/min }\end{array}$ & $\begin{array}{l}38,31,39,30,35,34,32,37,36 \\
17,36,47,53,62,68,75,80,85\end{array}$ & 731.49 \\
\hline
\end{tabular}

Then, the optimization characteristic of load restoration is investigated similarly. $\delta_{1}$ is assumed as $0, \delta_{2}$ is changed to determine the minimal requirement of weighted amount of load pick-up. The first time-step is taken as an example. The values of uncertainty radius $a_{2}$ and corresponding load restoration scheme are shown in Table 6.

Table 6. The load restoration scheme in first time-step considering double uncertainty.

\begin{tabular}{cccc}
\hline $\boldsymbol{\delta}_{\mathbf{2}}$ & $\boldsymbol{a}_{\mathbf{2}}$ & Load Restoration & $\begin{array}{c}\text { Amount of Load } \\
\text { Pick-Up/MW }\end{array}$ \\
\hline 0.2 & 0.13 & $8(2), 31(1), 18(1), 27(1)$ & 56.4 \\
0.4 & 0.25 & $4(2), 15(1), 27(1)$ & 42.5 \\
0.6 & 0.47 & $27(1), 27(2)$ & 28.0 \\
0.75 & 0.83 & $12(1), 31(1)$ & 17.7 \\
\hline
\end{tabular}

It can be seen that the load restoration schemes can be obtained through the optimization model that is proposed under different uncertainty radius $a_{2}$. The dispatcher can look up the table to select the appropriate load restoration scheme according to the approximate fluctuation range of load pick-up based on historical data. A comparison between load restoration scheme considering double uncertainty and that considering ideal situation is shown in Figure 7.

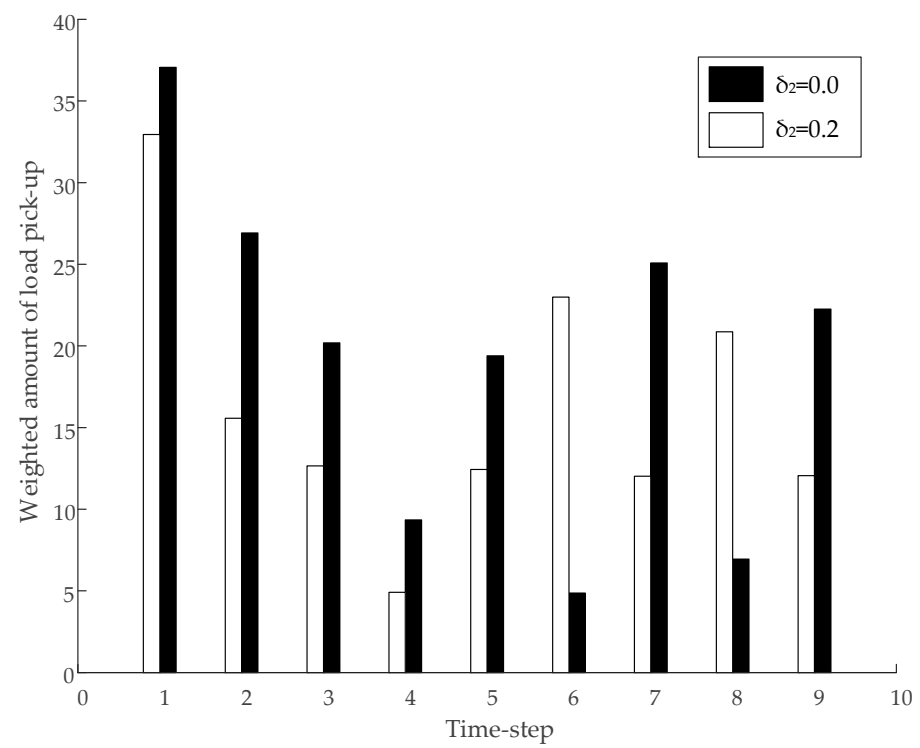

Figure 7. A comparison of load restoration scheme considering double uncertainty.

It can be seen from Figure 7 that except time-step 6 and 8, the weighted amount of load pick-up considering double uncertainty is lower than the ideal value not only in a single time-step, but also in the entire restoration process. The essence of IGDT is to sacrifice part of the load pick-up and enhance the robustness of the system restoration. 
Finally, the relationship between uncertainty radius and deviation factors is analyzed. $\delta_{1}-\alpha_{1}$ and $\delta_{2}-\alpha_{2}$ are shown in Figure 8.
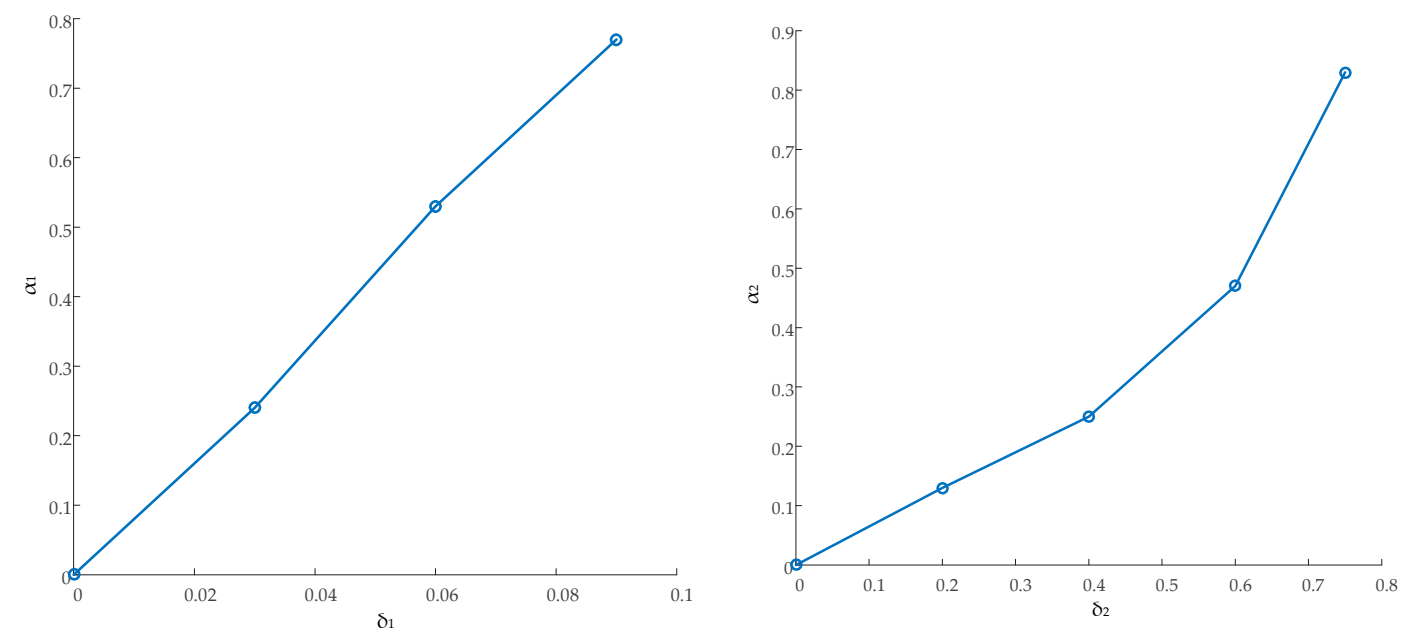

Figure 8. The relationship between uncertainty radius and deviation factors.

The deviation factor has a positive correlation with the maximum uncertainty radius. Specifically, the smaller requirement of generating output is, the longer the start-up time of a unit can be tolerated. The smaller requirement of weighted amount of load pick-up is, the greater fluctuation of load pick-up in each feeder can be tolerated.

\subsection{Comparison of Optimization Characteristics}

To further verify the advantages of the proposed source-load coordinated restoration method based on IGDT, the proposed method in this paper is compared to fuzzy chance constrained programming. Assuming that the start-up time of generating unit and the amount of load pick-up are fluctuated around the predicted value in $(0.8,1.2)$. Double uncertainty is expressed by triangular fuzzy parameters and confidence intervals are all assumed as 0.8 . The weighted amount of load pick-up in each time-step based on different methods is recorded, as shown in Figure 9. If the constraints are not satisfied, then the weighted amount of load pick-up is 0 .

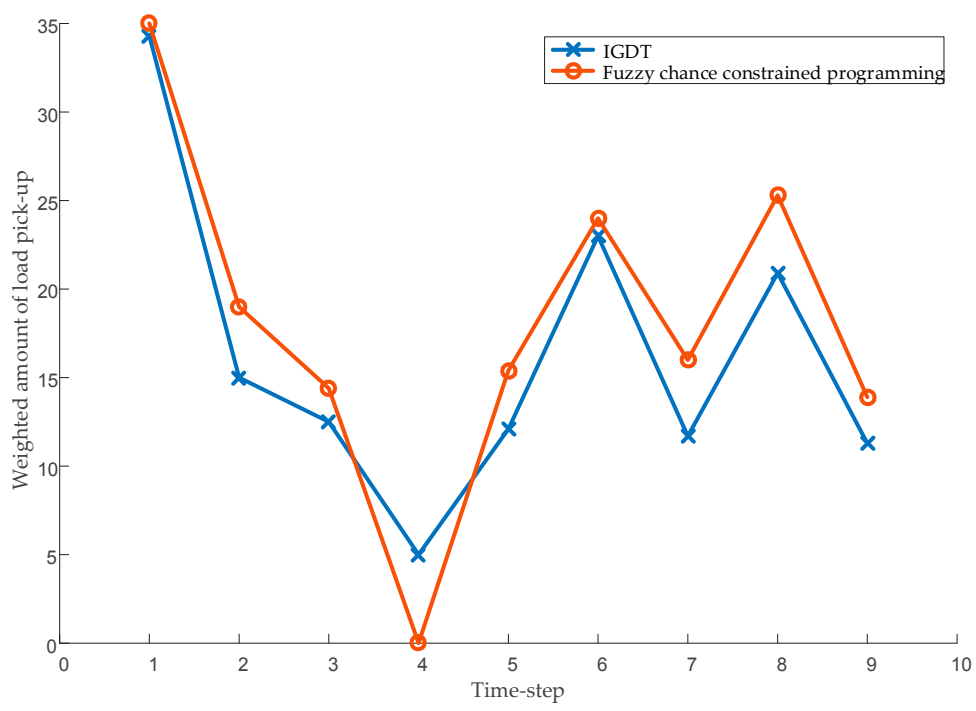

Figure 9. A comparison of load restoration scheme based on different methods. 
The source-load coordinated restoration scheme based on fuzzy chance constrained programming is shown in Table 7.

Table 7. The source-load coordinated restoration scheme based on fuzzy chance constrained programming.

\begin{tabular}{cccccc}
\hline Time-Step & Time/min & $\begin{array}{c}\text { Unit } \\
\text { Restoration }\end{array}$ & Load Restoration & $\begin{array}{c}\text { Amount of Load } \\
\text { Pick-Up/MW }\end{array}$ & $\begin{array}{c}\text { Frequency } \\
\text { Deviation/Hz }\end{array}$ \\
\hline 1 & $0-25$ & 31 & $8(2), 15(1), 18(1)$, & 62.8 & 0.486 \\
2 & $25-38$ & 32 & $27(1)$ & 35.6 & 0.320 \\
3 & $38-44$ & 30 & $16(1), 23(1)$ & 22.0 & 0.231 \\
4 & $44-56$ & 39 & $4(2), 24(2), 4(1)$, & 90.1 & 0.513 \\
5 & $56-60$ & 35 & $39(4), 12(1), 31(1)$ & 23.4 & 0.193 \\
6 & $60-66$ & 34 & $16(2), 4(4)$ & 37.4 & 0.297 \\
7 & $66-70$ & 37 & $39(5), 24(1)$ & 32.1 & 0.195 \\
8 & $70-75$ & 36 & $3(1), 15(2)$ & 39.0 & 0.223 \\
9 & $75-81$ & 38 & $21(1), 16(3), 4(3)$ & 38.1 & 0.204 \\
\hline
\end{tabular}

It can be seen that the source-load coordinated restoration scheme based on fuzzy chance constrained programming can not meet the security constraints in time-step 4 . The descending value of frequency is more than $0.5 \mathrm{~Hz}$, which exceeds the limit.

In summary, the source-load coordinated restoration method based on IGDT can tolerate the fluctuation of the start-up time of the generating unit and the amount of load pick-up. Although the weighted amount of load pick-up is smaller than fuzzy chance constrained programming, it is more robust in the overall restoration process.

\section{Conclusions}

An optimal source-load coordinated restoration model considering double uncertainty is proposed, in which unit and load are restored coordinately in the manner of different priorities. Firstly, double uncertainty including the start-up time of generating units and the amount of load pick-up is analyzed to build time-domain characteristics of unit and load. On this basis, a coupled multi-objective optimization model is established with uncertainties; the units to be restarted can be determined by maximizing the generating power output, while the loads to be restored can be determined by maximizing the weighted amount of load pick-up. Then, IGDT is adopted to convert uncertainty multi-objective optimization model to a certain one with robustness. NSGA-II is applied to solve the proposed model. Finally, The test results on the IEEE 39-bus system show that unit and load can be effectively and coordinately restored, which tolerates the most uncertainty and meet the minimal requirements. The proposed method improves the robustness of restoration and provides a useful way for decision making of the restoration scheme.

Acknowledgments: This work was supported by the Science and Technology Program of State Grid Jiangsu Branch (SGJS0000DKJS1601327).

Author Contributions: Ping Jiang is the principal investigator of this work. He conceived the experiments and wrote the manuscript; Qiwei Chen contributed to the experiments, data analysis work and language editing.

Conflicts of Interest: The authors declare no conflict of interest.

\section{References}

1. Li, C.; He, J.; Zhang, P.; Xu, Y. A Novel Sectionalizing Method for Power System Parallel Restoration Based on Minimum Spanning Tree. Energies 2017, 10, 948. [CrossRef]

2. Wang, J.; Mu, L.; Zhang, F.; Zhang, X. A Parallel Restoration for Black Start of Microgrids Considering Characteristics of Distributed Generation. Energies 2018, 11, 1. [CrossRef] 
3. Gu, X.; Zhong, H. Optimisation of Network Reconfiguration Based on A Two-layer Unit-restarting Framework for Power System Restoration. IET Gener. Transm. Distrib. 2012, 6, 693-700. [CrossRef]

4. Quiros-Tortos, J.; Panteli, M.; Wall, P.; Terzija, V. Sectionalising Methodology for Parallel System Restoration Based on Graph Theory. IET Gener. Transm. Distrib. 2015, 9, 1216-1225. [CrossRef]

5. Qin, Z.; Hou, Y.; Liu, C.-C.; Liu, S.; Sun, W. Coordinating Generation and Load Pickup during Load Restoration with Discrete Load Increments and Reserve Constraints. IET Gener. Transm. Distrib. 2015, 9, 2437-2446. [CrossRef]

6. Xu, Y.; Liu, W. Novel Multiagent Based Load Restoration Algorithm for Microgrids. IEEE Trans. Smart Grid 2011, 2, 152-161. [CrossRef]

7. Jiang, Y.; Chen, S.; Liu, C.; Sun, W.; Luo, X.; Liu, S.; Bhatt, N.; Uppalapati, S.; Forcum, D. Blackstart Capability Planning for Power System Restoration. Int. J. Electr. Power Energy Syst. 2016, 86, 127-137. [CrossRef]

8. Sun, W.; Liu, C.; Zhang, L. Optimal Generator Star-up Strategy for Bulk Power System Restoration. IEEE Trans. Power Syst. 2011, 26, 1357-1366. [CrossRef]

9. Nourizadeh, S.; Sarmadi, S.A.N.; Karimi, M.J.; Ranjbara, A.M. Power System Restoration Planning Based on Wide Area Measurement System. Int. J. Electr. Power Energy Syst. 2012, 43, 526-530. [CrossRef]

10. Gu, X.; Wang, D. Investigation on extended black-start schemes of power system considering reasonable load restoration. In Proceedings of the IEEE PES Asia-Pacific Power and Energy Engineering Conference, Hongkong, China, 7-10 December 2014; pp. 1-5.

11. Xu, Z.; Yang, P.; Zheng, Q.; Zeng, Z. Study on black start strategy of microgrids with PV and multiple energy storage systems. In Proceedings of the IEEE International Conference on Electrical Machines and Systems, Pattaya, Thailand, 25-28 October 2016; pp. 402-408.

12. Lin, Z.; Wen, F.; Huang, J.; Zhou, H. Evaluation of black-start schemes employing Entropy weight-based decision-making theory. J. Energy Eng. 2010, 136, 42-49. [CrossRef]

13. Escobedo, A.R.; Moreno-Centeno, E.; Hedman, K.W. Topology control for load shed recovery. IEEE Trans. Power Syst. 2014, 29, 908-916. [CrossRef]

14. Ye, H.; Liu, Y. A new method for standing phase angle reduction in system restoration by incorporating load pickup as a control means. Int. J. Electr. Power Energy Syst. 2013, 53, 664-674. [CrossRef]

15. Qu, H.; Liu, Y. Maximizing Restorable Load Amount for Specific Substation during System Restoration. Int. J. Electr. Power Energy Syst. 2012, 43, 1213-1220. [CrossRef]

16. Gholami, A.; Aminifar, F. A Hierarchical Response-Based Approach to the Load Restoration Problem. IEEE Trans. Smart Grid 2017, 8, 1700-1709. [CrossRef]

17. Liu, W.; Lin, Z.; Wen, F.; Ledwich, G. A Wide Area Monitoring System Based Load Restoration Method. IEEE Trans. Power Syst. 2013, 28, 2025-2034. [CrossRef]

18. Wang, D.; Gu, X.; Zhou, G.; Li, S.; Liang, H. Decision-making Optimization of Power System Extended Black-start Coordinating Unit Restoration with Load Restoration. Int. Trans. Electr. Energy Syst. 2017, 27, e2367. [CrossRef]

19. Wang, H.; Lin, Z.; Wen, F.; Ledwich, G.; Xue, Y.; Zhou, Y.; Huang, Y. Black-start Decision-making with Interval Representations of Uncertain Factors. Int. J. Electr. Power Energy Syst. 2016, 79, 34-41. [CrossRef]

20. Wang, B.; Li, Y.; Watada, J. Supply Reliability and Generation Cost Analysis Due to Load Forecast Uncertainty in Unit Commitment Problems. IEEE Trans. Power Syst. 2013, 28, 2242-2252. [CrossRef]

21. Chen, K.; Wu, W.; Zhang, B.; Sun, H. Robust Restoration Decision-making Model for Distribution Networks Based on Information Gap Decision Theory. IEEE Trans. Smart Grid 2015, 6, 587-597. [CrossRef]

22. Soroudi, A.; Ehsan, M. IGDT Based Robust Decision Making Tool for DNOs in Load Procurement Under Severe Uncertainty. IEEE Trans. Smart Grid 2013, 4, 886-895. [CrossRef]

23. Mohammadi-lvatloo, B.; Zareipour, H.; Amjady, N.; Ehsan, M. Application of Information-Gap Decision Theory to Risk-constrained Self-scheduling of GenCos. IEEE Trans. Power Syst. 2013, 28, 1093-1102. [CrossRef]

24. Adibi, M.M.; Borkoski, J.N.; Kafka, R.J.; Volkmann, T.L. Frequency Response of Prime Movers during Restoration. IEEE Trans. Power Syst. 1999, 14, 751-756. [CrossRef]

25. Pereira, L.A.; Haffner, S.; Nicol, G.; Dias, T.F. Multiobjective optimization of Five-phase Induction Machines Based on NSGA-II. IEEE Trans. Ind. Electr. 2017, 64, 9844-9853. [CrossRef] 
26. Haffner, S.; Pereira, L.A.; Pereira, L.F.A. A Method for Optimization of Five-phase Induction Machines Based on Genetic Algorithm. J. Control Autom. Electr. Syst. 2015, 26, 521-534. [CrossRef]

27. Deb, K.; Pratap, A.; Agarwal, S.; Meyarivan, T. A Fast and Elitist Multiobjective Genetic Algorithm: NSGA-II. IEEE Trans. Evolut. Comput. 2002, 6, 182-197. [CrossRef] 